\title{
Intracranial Venous and Mesenteric Vein Thrombosis in a Case of Lung Cancer with Brain, Bone, and Leptomeningeal Metastasis
}

\author{
Hongdan Shen, Xianhua Fu* \\ Department of Medical Oncology, The Second Affiliated Hospital, School of Medicine, Zhejiang University, Hangzhou, China \\ Email: *mcqueen@zju.edu.cn
}

How to cite this paper: Shen, H.D. and Fu, X.H. (2020) Intracranial Venous and Mesenteric Vein Thrombosis in a Case of Lung Cancer with Brain, Bone, and Leptomeningeal Metastasis. Case Reports in Clinical Medicine, 9, 1-6.

https://doi.org/10.4236/crcm.2020.91001

Received: December 8, 2019

Accepted: December 23, 2019

Published: December 26, 2019

Copyright $\odot 2020$ by author(s) and Scientific Research Publishing Inc. This work is licensed under the Creative Commons Attribution International License (CC BY 4.0).

http://creativecommons.org/licenses/by/4.0/

\begin{abstract}
Cancer patients with venous thromboembolism (VTE) are not rare, and the second death cause in patients with cancer is VTE. Although VTE itself is associated with reduced survival particularly in cancer patients, many oncologists may not place enough importance in positive prevention of VTE. Here, we report the case of a 38-year-old male lung cancer patient with brain, bone and leptomeningeal metastasis who diagnosed as a consequential intracranial venous and mesenteric vein thrombosis. The evolution of headache has not been noted gradually, from which led to the underestimation of headache and without careful consideration on the possibility of suspected intracranial venous thrombosis. The problem of mesenteric vein thrombosis reflects the dilemma resulted from the potential risk of thrombogenesis and the high risk of hemorrhage. Preventive anticoagulation strategies are key means especially in patients with cancer, and the management problems of this condition are discussed.
\end{abstract}

\section{Keywords}

Venous Thromboembolism, Thromboprophylaxis, Cancer Management

\section{Introduction}

Venous thromboembolism (VTE) mainly consists of deep venous thrombosis and pulmonary embolism. VTE risk for cancer patients could include the patients themselves factors and treatments-related factors such as surgery, radiotherapy and chemoradiotherapy. Hospitalized cancer patients are at increased risk for venous thromboembolism (VTE) [1]. Though prophylactic anticoagulants are recommended to reduce VTE and do not increase major bleeding in 
outpatients with cancer [2] [3]. The present clinical guidelines recommend primary VTE prophylaxis that still mismatch between the absolute VTE risk and demands of VTE prophylaxis for cancer patients. Now two common VTE risk assessment models have been recommended, which are respectively the Caprini and Padua risk assessment models [4]. Some literature supporting the Padua risk assessment models has been revealed to have a better stratification of the thromboembolic risk in hospitalized cancer patients [5] [6]. In the clinic, the loose use of Padua prediction score occurs frequently. Finally, this case appeals that vein thrombosis prophylaxis is essential components of comprehensive cancer management, provided our clinicians to weigh the risk for VTE and bleeding risk [7].

\section{Case}

A 38-years-old male smoker who smoked more than 10 years (mean 10 cigarettes/day) diagnosed as lung cancer by fibreoptic bronchoscopy in local hospital. He transferred to the second affiliated hospital of Zhejiang University School of Medicine and his pathological sections were re-evaluated by our experienced pathologist. Then infiltrative lung adenocarcinoma with EGFR L858R mutation was diagnosed. Radionuclide bone imaging showed bone destruction of lumbar vertebra (L1), then bone metastasis was first considered. It is of note that the patient was diagnosed as papillary thyroid carcinoma and received thyroidectomy for thyroid tumors six years ago. His father died because of esophageal cancer. The patient was treated with gefitinib and clinical benefit was evaluated bimonthly. Then, multiple micro-enhanced nodules in the brain parenchyma (Figure 2(A), Figure 2(B)) were found by head MRI enhanced tomography and considered as brain metastasis after treatment of gefitinib for total 11 months. And a second EGFR mutation test revealed EGFR Thr790M mutation, the abundance of Thr790Met-mutant was $0.2 \%$. So the patient was treated with whole brain radiotherapy (WBRT) and concurrent drug of AZD9291. On day 7 after WBRT the patients suffered with aggravated symptoms of insufferable headache and involuntary right extremity motor. On day 10 after WBRT the patient was sent to the emergency room on account of disturbance of consciousness. Increased CSF pressure $\left(>400 \mathrm{~mm} \mathrm{H}_{2} \mathrm{O}\right)$ was detected by the lumbar puncture. And a few atypical tumor cells were found in CSF from the cerebral ventricle puncture (Figure $2(F)$, Figure $2(G)$ ). A diagnosis of hydrocephalus, symptomatic epilepsy was made. Then multiple thrombosis located in superior sagittal sinus and superficial cerebral veins are shown in contrast-enhanced MR venography (CE-MRV, Figures 1(B)-(D)). 18F-FDG-PET/CT Scan showed remarkable increased glucose metabolism located in lung, bone, brain, lumbar vertebra (L3), and $4 \mathrm{R}$ region mediastinum lymph node (Figures $2(\mathrm{C})-(\mathrm{E})$ ). Low-molecular-weight heparin was initiated for VTE treatment. Mannitol, glycerin fructose, and the antiepileptic drugs were used to lower intracranial pressure and control the seizure. Then AZD9291 was replaced with erlotinib combined with anlotinibhydrochloride 

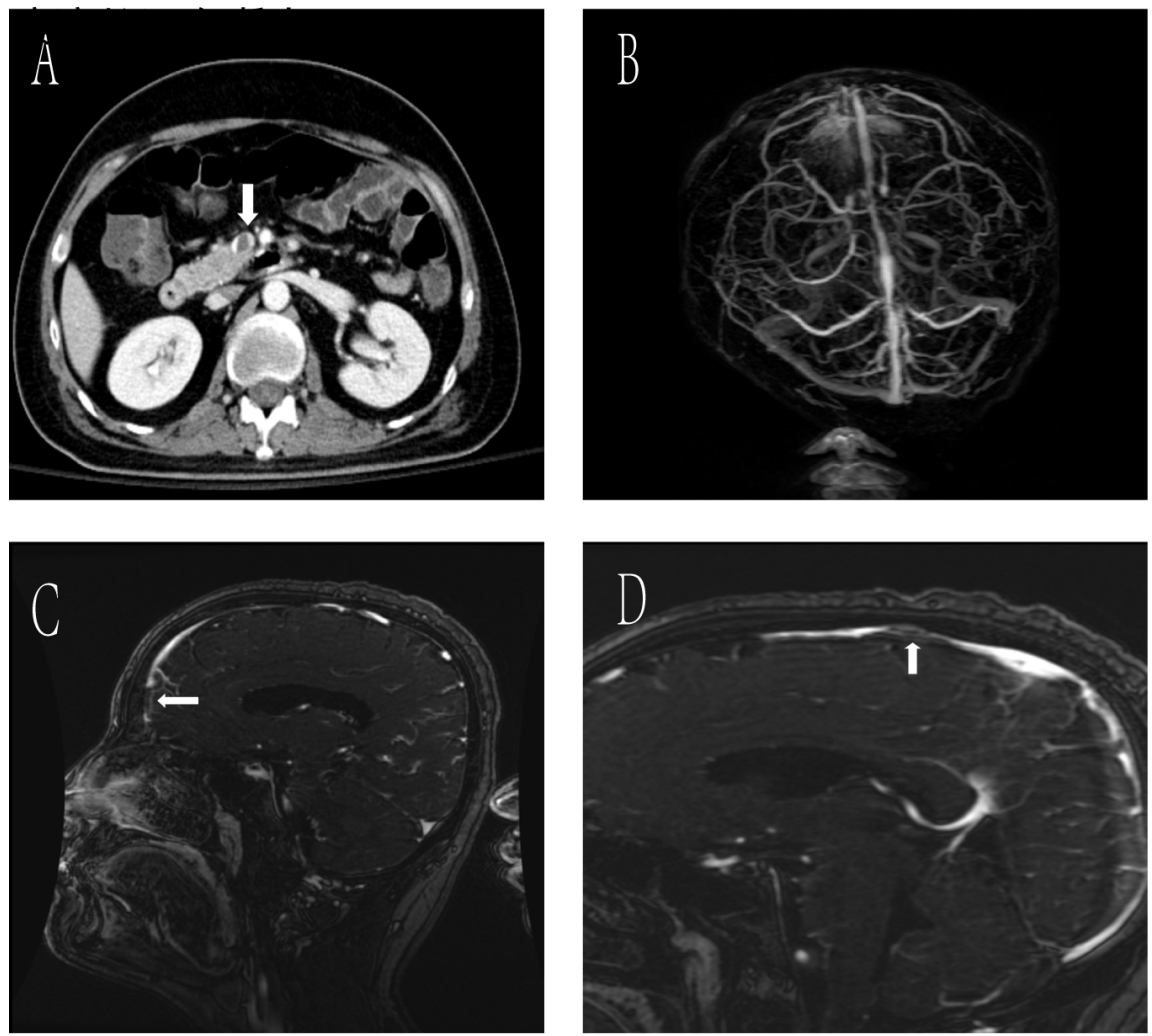

Figure 1. Images of the venous thromboembolism. (A) The abdominal contrast-enhanced CT shows an intraluminal filling defect located in the superior mesenteric vein (white arrow). (B, C, D) On CE-MRV, multiple thrombosis in left superficial cerebral veins (figure $B$ ); absence of normal flow signal in the region of superior sagittal sinus (figure C, D, white arrow)

because of severe gastrointestinal toxicity of AZD9291 one month later. The patient was managed with a multidisciplinary oncology consultation and advised to transfer with afatinib combined with bevacizumab. Unfortunately, a new onset of superior mesenteric vein thrombosis was found by the abdominal enhanced CT scan (Figure 1(A)). Curative efficacy was assessed as tumor progression regardless of replaced treatment regimens form five courses of afatinib combined with bevacizumab, paclitaxel combined with carboplatin and pembrolizumab for one course, AZD9291 combined with cabozantinib for three months, and pemetrexed combined with bevacizumab for one course. The patient died with an overall survival of nearly 2 years.

\section{Discussion}

Reviewing clinical history was that the patient appeared initial symptom of looming headache. Then head space occupying lesions were revealed by head MRI and considered as brain metastasis. Then the patient suffered aggravated headache and received WBRT. After WBRT, aggravated headache, symptomatic epilepsy and disturbance of consciousness occurred on this patient. Leptomeningeal metastasis was highly suspected due to atypical cells in CSF. Measuring 

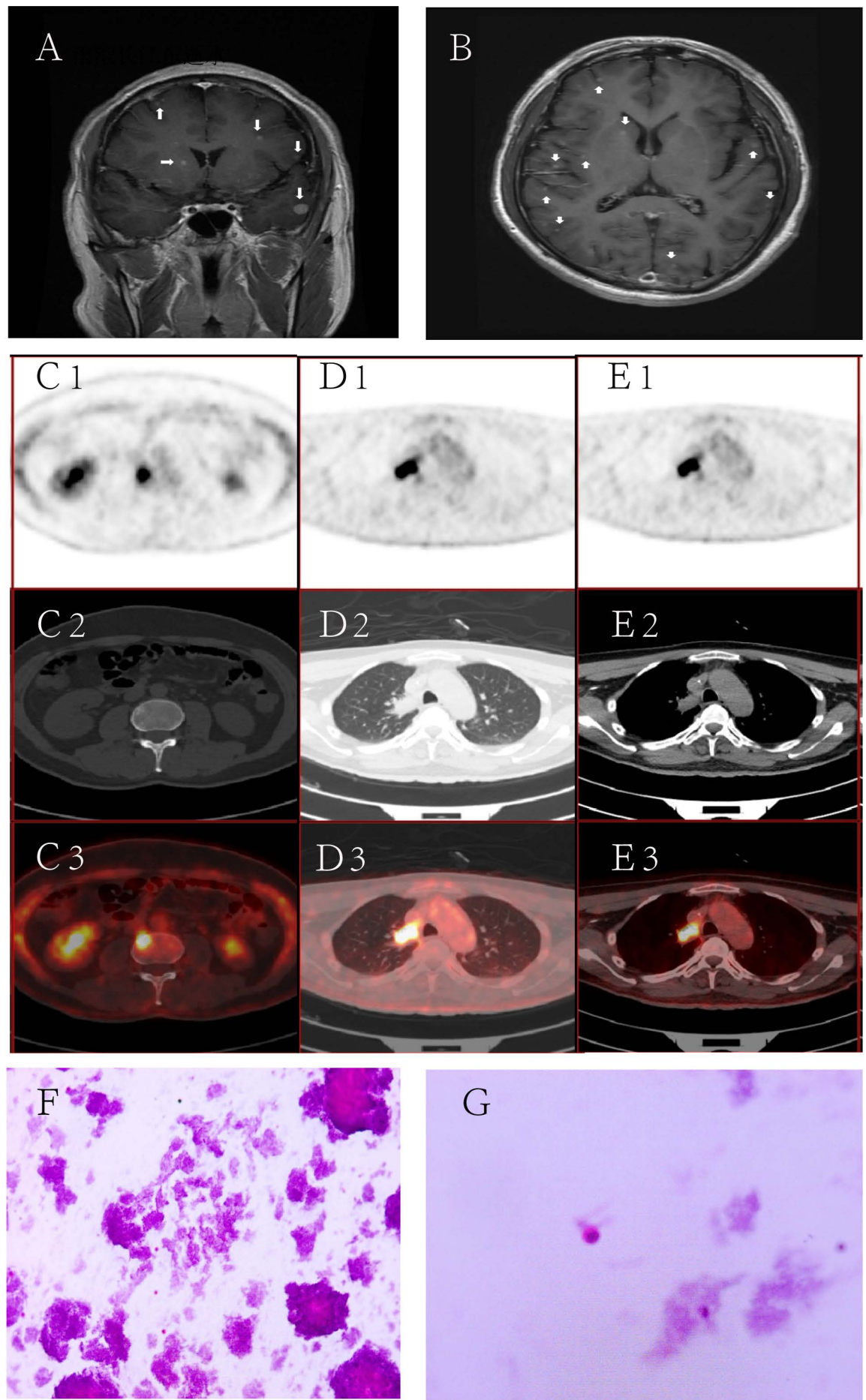

Figure 2. Systemic metastasis. (A, B) Cranial MRI: the abnormal brain signal intensity within bilateral cerebral parenchyma and cerebellum (white arrow), slightly large mass lesion in the left temporal lobe; (C1-C3) On PET-CT, increased FDG uptake in lumbar vertebra (L3), (SUV-max: 13.24); (D1-D3) On PET-CT, irregular and high-density mass with burrs around (size: $2.34 \times 1.52 \mathrm{~cm}$ ) located in the right superior apical segment (SUV-max: 20.69); (E1-E3) On PET-CT, increased FDG uptake in 4R region mediastinum lymph node (SUV-max: 10.73); (F): CSF cytology: a few scattered atypical cells were seen (magnification, $\times 200$ ); (G) CSF cytology: at higher magnification (magnification, $\times 400)$. 
lumbar puncture opening pressure certified high intracranial pressure. Collectively, with the progression of the disease, the symptoms from intracranial hypertension through focal neurological deficits suggest neuronal system abnormality. In hindsight, headache should also raise suspicion for intracranial venous thrombosis. This patient's comprehensive management is multi-factorial. Among them, optimal thromboprophylaxis is essential to reduce non-tumor-related morbidity and mortality [8]. In this patient, low-molecular-weight heparin (LMWH) was used actively as VTE treatment once diagnosed as intracranial venous thrombosis. There were many predisposing risk factors for VTE in this patient, including the continued presence of cancer or complication, but he was also at higher risk of bleeding due to chemoradiotherapy and targeted drugs-related adverse reactions such as anlotinib and bevacizumab. As a monoclonal antibody against vascular endothelial growth factor, bevacizumab is associated with a recognized possibility of increased risk of bleeding [9]. Management of VTE prophylaxis was challenging. Thromboprophylaxis is still underestimated by many oncologists [10]. Subsequently this patient stopped daily injection of LMWH and oral anticoagulants during bevacizumab therapy. Superior mesenteric vein thrombosis was found during abdominal contrast-enhanced CT evaluation after chemotherapy. In this case, clinical deterioration of disease resulted in brain degeneration, respiratory failure, and ultimately, death.

While poor prognosis associated with cancer, not an unexpected outcome, it is essential for oncologists to have a better understanding of cancer-associated thrombosis and the clinical complications. Thromboprophylaxis and the early diagnosis of venous thrombosis are required during management of the high-risk cancer patients with suspected venous thrombosis. More importantly, clinicians should fully take into account the needs of developing a well-planned therapeutic strategy on the basis of guiding anticoagulant strategies in selected patients. In cases of venous thrombosis in cancer patients, an early detection and treatment are required for a better prognosis.

\section{Acknowledgements}

We want to express our genuine gratitude to the patient's family for consenting to case publication.

\section{Conflicts of Interest}

The authors declare no conflicts of interest regarding the publication of this paper.

\section{References}

[1] Rossel, A., Robert-Ebadi, H., Combescure, C, et al. (2019) Anticoagulant Therapy for Acute Venous Thromboembolism in Cancer Patients: A Systematic Review and Network Meta-Analysis. PLoS ONE, 14, e0213940. https://doi.org/10.1371/journal.pone.0213940

[2] Hajdenberg, J. (2019) Review: In Patients with Cancer, Prophylactic Anticoagulants 
Reduce VTE and Do Not Increase Major Bleeding. Annals of Internal Medicine, 171, JC65. https://doi.org/10.7326/ACPJ201912170-065

[3] Key, N.S., Bohlke, K. and Falanga, A. (2019) Venous Thromboembolism Prophylaxis and Treatment in Patients with Cancer: ASCO Clinical Practice Guideline Update Summary. Journal of Oncology Practice, 15, 661-664. https://doi.org/10.1200/JOP.19.00368

[4] Liu, X., Liu, C., Chen, X., et al. (2016) Comparison between Caprini and Padua Risk Assessment Models for Hospitalized Medical Patients at Risk for Venous Thromboembolism: A Retrospective Study. Interactive Cardiovascular and Thoracic Surgery, 23, 538-543. https://doi.org/10.1093/icvts/ivw158

[5] Germini, F., Agnelli, G., Fedele, M., et al. (2016) Padua Prediction Score or Clinical Judgment for Decision Making on Antithrombotic Prophylaxis: A Quasi-Randomized Controlled Trial. Journal of Thrombosis and Thrombolysis, 42, 336-339.

https://doi.org/10.1007/s11239-016-1358-Z

[6] Adelborg, K., Horvth-Puhó, E., Sundbll, J., et al. (2018) Risk and Prognosis of Cancer after Upper-Extremity Deep Venous Thrombosis: Apopulation-Based Cohort Study. Thrombosis Research, 161, 106-110.

https://doi.org/10.1016/j.thromres.2017.11.017

[7] Wang, T., Zwicker, J.I., Ay, C., Pabinger, I., Falanga, A., Antic, D., Noble, S., Khorana, A.A., Carrier, M. and Meyer, G. (2019) The Use of Direct Oral Anticoagulants for Primary Thromboprophylaxis in Ambulatory Cancer Patients: Guidance from the SSC of the ISTH. Journal of Thrombosis and Haemostasis, 17, 1772-1778. https://doi.org/10.1111/jth.14564

[8] Donnellan, E. and Khorana, A.A. (2017) Cancer and Venous Thromboembolic Disease: A Review. The Oncologist, 22, 199-207.

https://doi.org/10.1634/theoncologist.2016-0214

[9] Mortimer, J., Zonder, H.B. and Pal, S.K. (2012) Lessons Learned from the Bevacizumab Experience. Cancer Control, 19, 309-316. https://doi.org/10.1177/107327481201900407

[10] Khalil, J., Bensaid, B., Elkacemi, H., et al. (2015) Venous Thromboembolism in Cancer Patients: An Underestimated Major Health Problem. World Journal of Surgical Oncology, 13, 204. https://doi.org/10.1186/s12957-015-0592-8 